\title{
The Econ-ARK and HARK: Open Source Tools for Computational Economics
}

\author{
Christopher D. Carrolll*, Alexander M. Kaufman ${ }^{\ddagger}$, Jacqueline L. Kazi ${ }^{* *}$, Nathan M. Palmer ${ }^{\S}$, Matthew N. White ${ }^{\mathbb{I}}$ \\ https://youtu.be/1ytEhrnwu6A
}

\begin{abstract}
The Economics Algorithmic Repository and toolKit (Econ-ARK) aims to become a focal resource for computational economics. Its first 'framework, the Heterogeneous Agent Resources and Toolkit (HARK), provides a modern, robust, transparent set of tools to solve a class of macroeconomic models whose usefulness has become increasingly apparent both for economic policy and for research purposes, but whose adoption has been limited because the existing literature derives from idiosyncratic, hand-crafted, and often impenetrable legacy code. We expect future Econ-ARK frameworks (e.g., for analysis of the transmission of beliefs through agents' social networks) will draw heavily on key elements of the existing HARK framework, including the API, the structure, and documentation standards.
\end{abstract}

Index Terms-Heterogeneous-Agent Resources toolKit, econ-ark, computational economics, economic modeling

Disclaimer: Views expressed herein do not necessarily reflect the views of the respective institutions that employ the respective authors.

\section{Introduction}

The Economics Algorithmic Repository and toolKit (Econ-ARK) is a modular programming framework for solving and estimating macroeconomic and macro-financial models in which economic agents can exhibit significant heterogeneity. ${ }^{1}$ Models with extensive heterogeneity among agents can be extremely useful for policy and research purposes. However, the most commonly published macroeconomic and macro-finance models have very limited heterogeneity or none at all, in large part because these are the only models that can be easily solved with existing toolkits such as DYNARE [Adjemian2011].

In contrast, models with extensive heterogeneity among agents have no central toolkit and must be solved in a bespoke way. This requires a significant investment of time and human capital before a researcher can produce usable work. This results in needless code duplication, increasing the chance for error and wasting valuable research time. The Econ-ARK project addresses these concerns by providing a set of well-documented code modules that

* Corresponding author: ccarroll@jhu.edu

II Johns Hopkins University

¥ Woodrow Wilson School of Public Policy

** Capital One

$\S$ Econ-ARK

II University of Delaware

Copyright $\odot 2018$ Christopher D. Carroll et al. This is an open-access article distributed under the terms of the Creative Commons Attribution License, which permits unrestricted use, distribution, and reproduction in any medium, provided the original author and source are credited. can be composed together to solve a range of heterogeneous-agent models. Methodological advances in the computational literature allow many types of models to be solved using similar approaches; the Econ-ARK project simply brings these pieces together in one place. HARK is written in Python 2.7, with a pull request underway at the time of this writing to make it fully compatible with both Python 2.7 and 3.6.

Academic research in statistics has standardized on the use of the ' $R$ ' modeling language for scholarly communication, and on a suite of tools and standards of practice (the use of R-markdown, e.g.) that allow statisticians to communicate their ideas easily to each other. Many other scholarly fields have similarly developed suites of tools that allow scholars to easily and transparently exchange quantitative ideas and computational results without anyone having to master idiosyncratic details of anyone else's hand-crafted computer code.

The only branch of economics in which anything similar has happened is representative agent (RA) macroeconomics, which (to some degree) has standardized on the use of the DYNARE [Adjemian2011] toolkit for solving representative agent dynamic stochastic general equilibrium models.

We face two primary challenges. The first is to develop a set of resources and examples and standards of practice for communication that are self-evidently a major improvement on the way economists exchange ideas now. The second is to persuade scholars to adopt those tools.

The Econ-ARK is the vehicle by which we hope to achieve these objectives. We have begun with the creation of a toolkit for heterogeneous agents (HA) macroeconomics, in part because that is a field where the need for improvement in standards of transparency, openness, and reproducibility is particularly manifested, and partly because it is a field where important progress seems particularly feasible. QuantEcon is the most similar project to Econ-ARK and makes use of open source coding tools. However, that project focuses largely on foundational material appropriate for an introductory graduate course on numeric methods in macroeconomics, whereas the Econ-ARK is geared toward the production of new research. ${ }^{2}$

The traditional approach in macroeconomics has been to assume that aggregate behavior can be understood by modeling the behavior of a single 'representative agent' -- the 'representative consumer' or 'representative firm'. HA macroeconomics instead starts by constructing models of the behavior of individual microeconomic agents (a firm or a consumer, e.g.) that match key facts (say, that some people are borrowers and others are 
savers) from the rich microeconomic evidence about the behavior and circumstances of such agents. With that solid foundation in place, macroeconomic outcomes are constructed by aggregating the behavior of the individual agents subject to sensible requirements on the characteristics of the aggregate (such as that the aggregate amount borrowed cannot exceed a function of the aggregate amount saved). For a broad review of representative agent and heterogeneous agents economic modeling, see the discussion by [Guvenen2011] and [Kirman1992]. More broadly, the branch of agent-based macroeconomics explores the issues of emergence and complexity. The interested reader is directed to the Handbooks of Computational Economics, Volumes 2 and 4: [Tesfatsion2006] and [Hommes2018]. The most recent volume in particular outlines similarities and differences between more traditional heterogeneous agents macroeconomics and so-called "agent-based methods," inspired from fields such as physics and ecology.

The Heterogeneous-Agent Resources toolKit (HARK) is a modular programming framework for solving, estimating, and simulating macroeconomic models with heterogeneous agents. Agents in HARK can be heterogeneous in a large number of ways, such as in wealth, income processes, preferences, or expectations. Models with heterogeneity among agents have proven to be increasingly useful for policy and research purposes.

For example, recent work by [Kaplan2018] has shown that changes in interest rates affect the economy in large part by reallocating income flows across different types of households rather than by causing every household to change their behavior in the same way. The latter implicitly occurs in a traditional rational expectations model, but may be misleading regarding the underlying channel of the effect. [Carroll2017a] shows that the response to fiscal policy (such as stimulus payments or tax cuts) depends crucially on how such payments are distributed across different groups. For example, an extension of unemployment benefits has a bigger effect on spending than a cut in the capital gains tax. [Geanakoplos2010] outlines how heterogeneity drives the leverage cycle, and [Geanakoplos2012] applies these insights to large-scale model of the housing and mortgage markets.

HA models of the kind described above have had a major intellectual impact over the past few years. But the literature remains small, and contributions have come mostly from a few small groups of researchers with close connections to each other. An excellent overview of this literature can be found in the most recent volume of the Handbooks of Computational Economics [Hommes2018] and works cited therein.

In large part, this reflects the formidable technical challenges involved in constructing such models. In each case cited above, the codebase underlying the results is the result of many years of construction of hand-crafted code that has not been meaningfully vetted by researchers outside of the core group of contributors. This is not because researchers have refused to share their code; instead, it is because the codebases are so large, so idiosyncratic, and (in many cases) so poorly documented and organized as to be nearly incomprehensible to anyone but the original authors and their collaborators. Researchers with no connections to the pioneering scholars have therefore faced an unpalatable choice between investing years of their time reinventing the wheel, or investing years of their time deciphering someone else's peculiar and idiosyncratic code.

Researchers who must review the scientific and technical code written by others are keenly aware that the time required to review and understand another's code can dwarf the time required to simply re-write the code from scratch (conditional on understanding the underlying concepts). This can be particularly important when multiple researchers may need to work on parts of the same codebase, either across time or distance.

The HARK project addresses these concerns by providing a set of well-documented code modules that can be combined to solve a range of heterogeneous-agent models. Methodological advances in the computational economics literature allow many types of models to be solved using similar approaches; the key for HARK is to identify methodologies that are "modular" (in a sense to be described below).

In addition to these methodological advances, the HARK project adopts modern software development practices to ease the burden of code development, code review, code sharing, and collaboration for researchers dealing with computational methods.

Because these problems are generic (and not specific to computational economics), the software development community, and particularly the open-source community, has spent decades developing tools for programmers to quickly consume and understand code written by others, verify that it is correct, and to contribute back to a large and diverse codebase without fear of introducing bugs. The tools used by these professional developers include formal code documentation, unit testing structures, modern versioning systems for automatically tracking changes to code and content, and low-cost systems of communicating ideas, such as interactive programming notebooks that combine formatted mathematics with executable code and descriptive content. These tools operate particularly well in concert with one another, constituting an environment that can greatly accelerate project development for both individuals and collaborative teams. These technical tools are not new-- the HARK project simply aims to apply the best of them to the development of code in computational economics in order to increase researcher productivity, particularly when interacting with other researchers' code.

The rest of this paper will first outline the useful concepts we adopt from software development, with examples of each, and then demonstrate how these concepts are applied in turn to the key solution and estimation methods required to solve heterogeneousagent models. The sections are organized as follows: Section 1 discusses the natural modular structure of the types of problems HARK solves and provides an overview of the code structure that implements these solutions. Section 2 provides details of the core code modules in HARK. Section 3 outlines two examples that illustrate models in the HARK framework. Section 4 summarizes and concludes.

\section{HARK Structure}

The class of problems that HARK solves is highly modular by construction. There are approximately these steps in solving a rational heterogeneous agents model:

1) Specify the problem faced by an individual agent

2) Specify how the actions and states of individual agents collectively generate aggregate outcomes or processes

3) For given beliefs about aggregate processes, solve the individual agent's problem

4) Simulate the behavior of agents, generating a "history" of aggregate outcomes

5) Formulate new beliefs about the aggregate processes based on that history 
6) Iterate on steps 3-5 until beliefs converge

In isolation, steps 1 and 3 constitute the solution to a "microeconomic" model in HARK: how an individual agent should optimally act, treating all inputs to his problem as fixed. The inclusion of steps 2, 4, 5, and 6 embeds the microeconomic model in a "macroeconomic" model, requiring consistency among agents' individual behavior, the outcomes that result from the aggregation of these choices, and agents' beliefs about aggregate processes. The assumption of rationality is imposed by having the beliefs formulated in step 5 be justified given the history of aggregate outcomes; agents correctly interpret (a hypothetical) history when forming their new beliefs. Economists call such a solution a "rational expectations equilibrium", as agents' expectations are fulfilled by reality, and they have no reason to update these expectations or beliefs. ${ }^{3}$

In the section below titled "Sample Model: Perfect Foresight Consumption-Saving," we directly illustrate a microeconomic model in HARK; a full example of a macroeconomic model is outlined in [Carroll2017b].

To estimate a model for some research purpose, the economist tries to find the "deep" or "structural" parameters that make model outcomes best match particular features of some dataset. That is, the model is mathematically specified in steps 1 and 2 above, but the economist does not know the values of some vector of model parameters; the objective of the estimation is to find the parameters that make the model best "match" real data. As the dataset, features or moments to match, and particular estimation method (e.g. simulated method of moments or maximum likelihood estimation) are idiosyncratic to each research project, we will not elaborate further here.

In HARK, each of the solution steps is highly modular, and the structure of the solution method suggests a natural division of the code. (The solution method is dynamic programming and fixed point iteration, and the estimation method is Simulated Method of Moments. These are described in detail in [Carroll2012].)

Python modules in HARK can generally be categorized into three types: tools, models, and applications. Tool modules contain functions and classes with general purpose tools that have no inherent "economic content," but that can be used in many economic models as building blocks or utilities. Tools might include functions for data analysis (e.g. calculating Lorenz shares from data, or constructing a non-parametric kernel regression), functions to create and manipulate discrete approximations to continuous distributions, or classes for constructing interpolated approximations to non-parametric functions. Tool modules reside in the "top level" of HARK and have names like HARK.simulation and HARK. interpolation. The core functionality of HARK is in the tools modules; these will be discussed in detail in the following section.

Model modules specify particular economic models, including classes to represent agents in the model and the "market structure" in which they interact, and functions for solving the "one period problem" of those models. For example, Cons IndShockModel. py concerns consumption-saving models in which agents have CRRA utility over consumption and face idiosyncratic (Individual) shocks to permanent and transitory income. The module includes classes for representing "types" of consumers, along with functions for solving (several flavors of) the one period consumption-saving problem. When run, model modules might demonstrate example specifications of their models, filling in the model parameters with arbitrary values. When Cons IndShockModel.py is run, it specifies an infinite horizon consumer with a particular discount factor, permanent income growth rate, coefficient of relative risk aversion and other parameters, who faces lognormal shocks to permanent and transitory income each period with a particular standard deviation; it then solves this consumer's problem and graphically displays the results. ${ }^{4}$ Model modules generally have Model in their name. There are two broad types of models solved by HARK, "microeconomic" models and aggregate or "macroeconomic" models. In a microeconomic problem, agents solve their problem taking their environment as a given -- the "macro" environment is fixed exogenously. A macroeconomic problem is typically composed of a number of agents solving their own microeconomic problems, whose interactions affect the macroeconomic environment. Thus the aggregate processes that describe the agents' environment is endogenous to the individual-level decisions made by each agent. The two examples illustrate this in the "microeconomic" and "macroeconomic" sections below.

Application modules use tool and model modules to solve, simulate, and/or estimate economic models for a particular purpose. While tool modules have no particular economic content and model modules describe entire classes of economic models, applications are uses of a model for some research purpose. For example, /SolvingMicroDSOPs/StructEstimation.py uses a consumption-saving model from ConsIndShockModel.py, calibrating it with age-dependent sequences of permanent income growth, survival probabilities, and the standard deviation of income shocks (etc); it then estimates the coefficient of relative risk aversion and shifter for an age-varying sequence of discount factors that best fits simulated wealth profiles to empirical data from the Survey of Consumer Finance. A particular application might have multiple modules associated with it, all of which generally reside in one directory. Particular application modules will not be discussed in this paper further; please see the GitHub page and associated documentation for references to the application modules.

\section{Tool Modules}

HARK's root directory contains the following tool modules, each containing a variety of functions and classes that can be used in many economic models, or even for mathematical purposes that have nothing to do with economics. We expect that all of these modules will grow considerably in the near future, as new tools are "low hanging fruit" for contribution to the project.

\section{HARK.core}

This module contains core classes used by the rest of the HARK ecosystem. A key goal of the project is to create modularity and interoperability between models, making them easy to combine, adapt, and extend. To this end, the HARK. core module specifies a framework for economic models in HARK, creating a common structure for them on two levels that can be called "microeconomic" and "macroeconomic".

Beyond the model frameworks, HARK.core also defines a "superclass" called HARKobject. When solving a dynamic economic model, it is often required to consider whether two solutions are sufficiently close to each other to warrant stopping the process (i.e. approximate convergence). HARK specifies that classes should have a distance method that takes a single 
input and returns a non-negative value representing the (generally dimensionless) distance between the object in question and the input to the method. As a convenient default, HARKobject provides a "universal distance metric" that should be useful in many contexts. ${ }^{5}$ When defining a new subclass of HARKob ject, the user simply defines the attribute distance_criteria as a list of strings naming the attributes of the class that should be compared when calculating the distance between two instances of that class. See here for online documentation.

\section{HARK.utilities}

The HARK.utilities module carries a double meaning in its name, as it contains both utility functions (and their derivatives, inverses, and combinations thereof) in the economic modeling sense as well as utilities in the sense of general tools. Utility functions include constant relative risk aversion (CRRA) and constant absolute risk aversion (CARA). Other functions in HARK. utilities include data manipulation tools, functions for constructing discrete state space grids, and basic plotting tools. The module also includes functions for constructing discrete approximations to continuous distributions and manipulating these representations.

\section{HARK.interpolation}

The HARK.interpolation module defines classes for representing interpolated function approximations. Interpolation methods in HARK all inherit from a superclass such as HARKinterpolator1D or HARKinterpolator2D, wrapper classes that ensure interoperability across interpolation methods. These classes all inherit from HARKobject, so that they come equipped with the default distance metric. ${ }^{6}$

HARK.simulation: The HARK.simulation module provides tools for generating simulated data or shocks for postsolution use of models. Currently implemented distributions include normal, lognormal, Weibull (including exponential), uniform, Bernoulli, and discrete.

HARK.estimation: Methods for optimizing an objective function for the purposes of estimating a model can be found in HARK. estimation. As of this writing, the implementation includes minimization by the Nelder-Mead simplex method, minimization by a derivative-free Powell method variant, and two tools for resampling data (e.g., for a bootstrap). Future functionality will include global search methods, including genetic algorithms, simulated annealing, and differential evolution.

\section{Model Modules}

Microeconomic models in HARK use the Agent Type class to represent agents with an intertemporal optimization problem. Each of these models specifies a subclass of Agent Type; an instance of the subclass represents agents who are ex-ante homogeneous (they have common values for all parameters that describe the problem, such as risk aversion). The Agent Type class has a solve method that acts as a "universal microeconomic solver" for any properly formatted model, making it easier to set up a new model and to combine elements from different models; the solver is intended to encompass any model that can be framed as a sequence of one period problems. ${ }^{7}$

Macroeconomic models in HARK use the Market class to represent a market or other mechanisms by which agents' (i.e. instances of Agent Type subclasses) interactions are aggregated to produce "macro-level" outcomes. For example, the market in a consumption-saving model might combine the individual asset holdings of all agents in the market to generate aggregate savings and capital in the economy, which in turn produces the interest rate that agents care about. Agents then learn the aggregate capital level and interest rate, which affects their future actions. In this way, objects that microeconomic agents treat as exogenous when solving their individual-level problems (such as the interest rate) are made endogenous at at the macroeconomic level through the Market aggregator. Like Agent Type, the Market class also has a solve method, which seeks out a dynamic general equilibrium rule governing the aggregate processes.

\section{Microeconomics: the AgentType Class}

The core of our microeconomic dynamic optimization framework is a flexible object-oriented representation of economic agents. Each microeconomic model defines a subclass of AgentType, specifying additional model-specific features and methods while inheriting the methods of the superclass. This section provides a brief example of a problem solved by a microeconomic instance of Agent Type.

Sample Model: Perfect Foresight Consumption-Saving: To provide a concrete example of how the AgentType class works, consider the very simple case of a perfect foresight consumptionsaving model. The agent has time-separable, additive CRRA preferences over consumption $C_{t}$, discounting future utility at a constant rate. He receives a particular stream of labor income $Y_{t}$ each period and knows the interest rate $R$ on assets $A_{t}$ that he holds from one period to the next. His decision about how much to consume $C_{t}$ in a particular period out of total market resources $M_{t}$ can be expressed in Bellman form as:

$$
\begin{aligned}
V_{t}\left(M_{t}\right) & =\max _{C_{t}} \mathrm{u}\left(C_{t}\right)+\beta\left(1-D_{t+1}\right) E\left[V_{t+1}\left(M_{t+1}\right)\right] \\
A_{t} & =M_{t}-C_{t} \\
M_{t+1} & =R A_{t}+Y_{t+1} \\
Y_{t+1} & =\Gamma_{t+1} Y_{t} \\
\mathrm{u}(C) & =\frac{C^{1-\rho}}{1-\rho}
\end{aligned}
$$

The agent's problem is thus characterized by values of $\rho, R$, and $\beta$, plus sequences of survival probabilities $\left(1-D_{t+1}\right)$ and income growth factors $\Gamma_{t+1}$ for $t=0, \ldots, T-1$. This problem has an analytical solution for both the value function and the consumption function.

The ConsindShockModel.py module defines the class PerfForesightConsumertype as a subclass of Agent Type and provides solver classes for several variations of a consumption-saving model, including the perfect foresight problem. A HARK user could specify and solve a ten period perfect foresight model with the following two commands (the first command is split over multiple lines) :

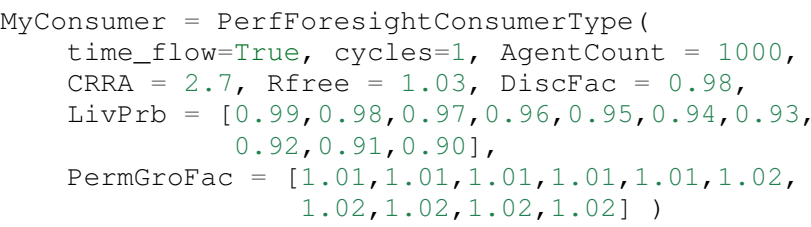

MyConsumer.solve()

The first line makes a new instance of ConsumerType, specifies that time is currently "flowing" forward, specifies that the se- 


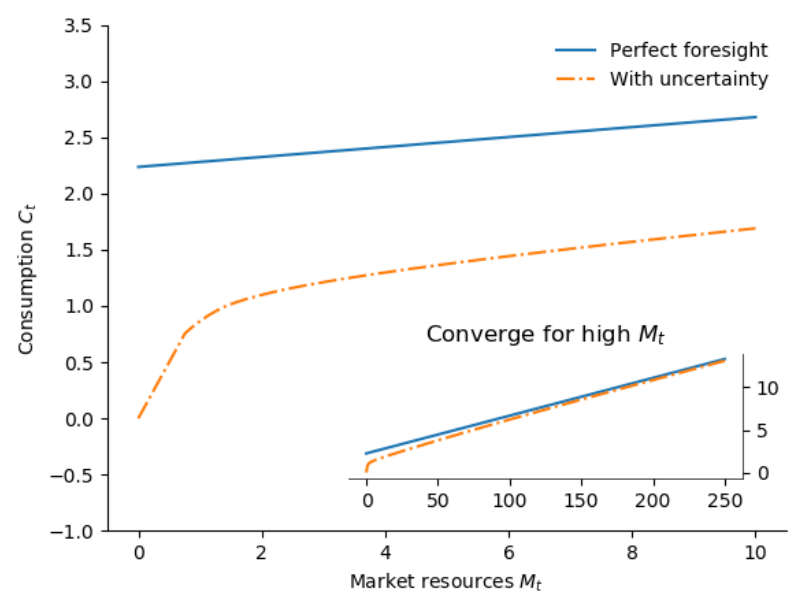

Fig. 1: Consumption Functions

quence of periods happens exactly once, and that, if the model is simulated after it is solved, there are 1000 agents with these exact characteristics. The next five lines (all part of the same command) set the time-invariant (CRRA is $\rho$, Rfree is $R$, and DiscFac is $\beta$ ) and time-varying parameters (LivPrb is $\left(1-D_{t+1}\right)$, PermGrofac is $\left.\Gamma_{t+1}\right)$. After running the solve method, MyConsumer will have an attribute called solution, which will be a list with eleven ConsumerSolution objects, representing the period-by-period solution to the model. ${ }^{8}$

The consumption function for a perfect foresight consumer is a linear function of market resources-- not terribly exciting. The marginal propensity to consume out of wealth doesn't change whether the consumer is rich or poor. When facing uncertain income, however, the consumption function is concave: the marginal propensity to consume is very high when agents are poor, and lower when they are rich. Moreover, agents facing income risk save more than agents under certainty. However, as agents facing uncertainty get richer, their consumption function converges to the perfect foresight consumption function-- rich but uncertain agents act like agents who face no income risk. In Figure 1, the solid blue line is consumption under certainty, while the dashed orange line is consumption under uncertainty. The inset plot demonstrates that these two functions converge as the horizontal axis of this plot is extended.

\section{Macroeconomics: the Market Class}

The modeling framework of Agent Type is called "microeconomic" because it pertains only to the dynamic optimization problem of individual agents, treating all inputs of the problem from their environment as exogenously fixed. In what we label as "macroeconomic" models, some of the inputs for the microeconomic models are endogenously determined by the collective states and choices of other agents in the model. In a rational dynamic general equilibrium, there must be consistency between agents' beliefs about these macroeconomic objects, their individual behavior, and the realizations of the macroeconomic objects or processes that result from individual choices.

The Market class in HARK. core provides a framework for such macroeconomic models, with a solve method that searches for a rational dynamic general equilibrium. An instance of Market includes as an attribute a list of Agent Type objects that compose the economy, a method for transforming microeconomic outcomes (states, controls, and/or shocks) into macroeconomic outcomes, and a method for interpreting a history or sequence of macroeconomic outcomes into a new "dynamic rule" for agents to believe. Agents treat the dynamic rule as an input to their microeconomic problem, conditioning their optimal policy functions on it. A dynamic general equilibrium is a fixed point dynamic rule: when agents act optimally while believing the equilibrium rule, their individual actions generate a macroeconomic history consistent with the equilibrium rule.

Down on the Farm: The Market class uses a farming metaphor to conceptualize the process for generating a history of macroeconomic outcomes in a model. Suppose all Agent Type agents in the economy believe in some dynamic rule (i.e. the rule is stored as attributes of each Agent Type, which directly or indirectly enters their dynamic optimization problem), and that they have each found the solution to their microeconomic model using their solve method. Further, the macroeconomic and microeconomic states have been reset to some initial orientation.

To generate a history of macroeconomic outcomes, the Market repeatedly loops over the following steps a set number of times:

1) sow: Distribute the macroeconomic state variables to all Agent Types in the market.

2) cultivate: Each AgentType executes their marketAction method, often corresponding to simulating one period of the microeconomic model.

3) reap: Microeconomic outcomes are gathered from each Agent Type in the market.

4) mill: Data gathered by reap is processed into new macroeconomic states according to some "aggregate market process".

5) store: Relevant macroeconomic states are added to a running history of outcomes.

This procedure is conducted by the makeHistory method of Market as a subroutine of its solve method. After making histories of the relevant macroeconomic variables, the market then executes its calcDynamics function with the macroeconomic history as inputs, generating a new dynamic rule to distribute to the Agent Type agents in the market. The process then begins again, with the agents solving their updated microeconomic models given the new dynamic rule; the solve loop continues until the "distance" between successive dynamic rules is sufficiently small.

Each subclass of Market has its own mill and calcDynamics methods, and designates which variables are to be gathered reap and distributed by sow, thus specifying what it means to generate "aggregate outcomes" and "form beliefs" in that particular model. We believe that the Market framework is general enough to encompass a very wide range of disparate models, from standard models in which individual assets are aggregated into productive capital, to models of choice over health insurance contracts with adverse selection and moral hazard, to models of direct agent-to-agent interaction more commonly seen in other scientific fields.

\section{Summary and Conclusion}

The Econ-ARK project's broadest aim is to provide a platform for improving communication and collaboration among economists on technical and computational questions. Its first framework, the 
HARK project, is a modular code library for constructing microeconomic and macroeconomic models with agents who differ from each other in serious ways: in dimensions whose consequences cannot be captured by analyzing the behavior of a single agent with average characteristics.

The HARK project is the starting point because it is an area where both the need and opportunities for improvement are great. In particular, existing code to solve HA models tends to be bespoke and idiosyncratic, with the consequence that tools are often reinvented by different researchers working on similar problems. Researchers should spend their valuable time producing research, not reinventing wheels. The HARK toolkit already provides a useful set of industrial strength, reliable, reusable wheels, constructed using a simple and easily extensible framework with clear documentation and testing regimens.

Part of the reason we are confident our goal is feasible is that the tools now available - Python, GitHub, and Jupyter notebooks among them - have finally reached a stage of maturity that can handle the communication of almost any message an economist might want to convey. ${ }^{9}$

The longer-term goals of the Econ-ARK project are to create a collaborative codebase that can serve the entire discipline of economics, employing the best of modern software development tools to accelerate understanding and implementation of cutting edge research tools. The solution methods employed in HARK are not the only methods available, and those who have additional methodological suggestions are strongly encouraged to contribute. The interested user should check the Econ-ARK GitHub page, particularly the HARK sub-page. There you will find a README and documentation. For the interested contributor, the issues page outlines the future improvements in progress. Issues labeled with "help wanted" are particularly good for getting started with contributing.

\section{Acknowledgements}

The Econ-ARK project is supported by a generous grant from the Alfred P. Sloan Foundation, with fiscal sponsorship from NumFOCUS. The authors would like to thank both organizations for their time, resources, and expertise.

\section{Bibliography}

[Adjemian2011] Adjemian, Stephane, Houtan Bastani, Michel Juillard, Ferhat Mihoubi, George Perendia, Marco Ratto, and Sebastien Villemot. 2011. "Dynare: Reference Manual, Version 4." Dynare Working Papers 1, CEPREMAP. RePEc: cpmdynare/001 .

[Carroll2012] Carroll, Christopher. 2012. "Solving Microeconomic Dynamic Stochastic Optimization Problems." Lecture Notes, Johns Hopkins University. url

[Carroll2017a] Carroll, Christopher, Jiri Slacalek, Kiichi Tokuoka, and Matthew N White. 2017. "The Distribution of Wealth and the Marginal Propensity to Consume." Quantitative Economics 8 (3). Wiley Online Library: 977-1020. doi:10.3982/QE694

[Carroll2017b] Carroll, Christopher, Alexander Kaufman, David Low, Nathan Palmer, and Matthew White. 2017. "A User's Guide for Hark: Heterogeneous Agents Resources and toolKit." Econ ARK. url

[Geanakoplos2010] Geanakoplos, John. 2010. "The Leverage Cycle." NBER Macroeconomics Annual 24 (1). The University of Chicago Press: 1-66. doi:10.1086/648285
[Geanakoplos2012] Geanakoplos, John, Robert Axtell, J Doyne Farmer, Peter Howitt, Benjamin Conlee, Jonathan Goldstein, Matthew Hendrey, Nathan M. Palmer, and Chun-Yi Yang. 2012. "Getting at Systemic Risk via an Agent-Based Model of the Housing Market." American Economic Review 102 (3): 53-58. doi:10.1257/aer.102.3.53

[Guvenen2011] Guvenen, Fatih. 2011. "Macroeconomics with Heterogeneity: A Practical Guide," Economic Quarterly, Federal Reserve Bank of Richmond 97 (3): 255-326. doi:10.3386/w17622

[Hommes2018] Hommes, Cars, and Blake LeBaron, eds. 2018. "Handbook of Computational Economics, Vol 4: Heterogeneous Agent Modeling," Handbook of Computational Economics, Elsevier, Vol 4: 2-796. doi:10.1016/S1574-0021(18)30018-2

[Kaplan2018] Kaplan, Greg, Benjamin Moll, and Giovanni L. Violante. 2018. "Monetary Policy According to HANK." American Economic Review 108 (3): 697-743. doi:10.1257/aer.20160042

[Kirman1992] Kirman, Alan P. 1992. "Whom or What Does the Representative Individual Represent?" Journal of Economic Perspectives 6 (2): 117-136. doi:10.1257/jep.6.2.117

[Tesfatsion2006] Tesfatsion, Leigh, Kenneth L. Judd, eds. 2006. "Handbook of Computational Economics, Vol 2: AgentBased Computational Economics," Handbook of Computational Economics, Elsevier, Vol 2: 829-1660. doi:10.1016/S15740021(05)02039-3

1. In this context, "heterogeneity" refers to both ex post heterogeneity-agents attaining different states or making different choices because they have experienced different random shocks in the model-- and ex ante heterogeneity- agents differing in their preferences, beliefs, or other innate attribute before the model "begins".

2. It is possible that some of the foundational tools from QuantEcon could be incorporated into the Econ-ARK, with the permission of its project leads. Our teams are in communication, and their advice has been valuable.

3. HARK does not impose the assumption of rationality; we use it here for exposition because it is the standard assumption in economics. The modular structure of the toolkit makes it easy to remove this assumption by, e.g., having agents misperceive their own problem, imperfectly process information, or form beliefs about aggregate processes that are not "justified" by the history.

4. Running Cons IndShockModel.py also demonstrates other variations of the consumption-saving problem, but their description is omitted here for brevity.

5. Roughly speaking, the universal distance metric is a recursive supnorm, returning the largest distance between two instances, among attributes named in distance_criteria. Those attributes might be complex objects themselves rather than real numbers, generating a recursive call to the universal distance metric.

6. Interpolation methods currently implemented in HARK include (multi)linear interpolation up to 4D, 1D cubic spline interpolation, 2D curvilinear interpolation over irregular grids, a 1D "lower envelope" interpolator, and others.

7. See [Carroll2017b] for a much more thorough discussion.

8. The solution to a dynamic optimal control problem is a set of policy functions and a value function, for each period. The policy function for this consumption-saving problem is how much to consume $C_{t}$ for a given amount of market resources $M_{t}$. The eleventh and final element of solution represents the trivial solution to the terminal period of the problem. For a much more detailed discussion, please see [Carroll2017b].

9. See the recent blog post by Paul Romer, "Jupyter, Mathematica, and the Future of the Research Paper" for a fuller argument). 